\title{
Ss \\ Two particle model for studying the effects of space-charge force on strong head-tail instabilities
}

\author{
Yong Ho Chin \\ KEK High Energy Accelerator Research Organization, 1-1 Oho, Tsukuba, Ibaraki-ken, 305-0801, Japan \\ Alexander Wu Chao \\ SLAC Stanford Linear Accelerator Center, 2575 Sand Hill Road, Menlo Park, California 94025, USA
}

Michael M. Blaskiewicz

BNL Brookhaven National Laboratory, P.O. Box 5000, Upton, New York 11973-5000, USA

(Received 12 May 2015; published 19 January 2016)

\begin{abstract}
In this paper, we present a new two particle model for studying the strong head-tail instabilities in the presence of the space-charge force. It is a simple expansion of the well-known two particle model for strong head-tail instability and is still analytically solvable. No chromaticity effect is included. It leads to a formula for the growth rate as a function of the two dimensionless parameters: the space-charge tune shift parameter (normalized by the synchrotron tune) and the wakefield strength, $\Upsilon$. The three-dimensional contour plot of the growth rate as a function of those two dimensionless parameters reveals stopband structures. Many simulation results generally indicate that a strong head-tail instability can be damped by a weak spacecharge force, but the beam becomes unstable again when the space-charge force is further increased. The new two particle model indicates a similar behavior. In weak space-charge regions, additional tune shifts by the space-charge force dissolve the mode coupling. As the space-charge force is increased, they conversely restore the mode coupling, but then a further increase of the space-charge force decouples the modes again. This mode coupling/decoupling behavior creates the stopband structures.
\end{abstract}

DOI: 10.1103/PhysRevAccelBeams.19.014201

\section{INTRODUCTION}

In low energy high-intensity hadron machines, the spacecharge tune shift is an important parameter in the design and operation of the machines. The space-charge force is also believed to affect the behavior of beam instabilities. Many theoretical and simulation studies have been made for a better understanding of their interplay [1-9]. They generally indicate that beam instability can be damped when the space-charge force is weak, but the beam becomes unstable again when it becomes too strong (for example, see Fig. 1 of Ref [1] and Fig. 10 of Ref. [7]). The mechanism of this loss of the damping effect due to a strong space-charge force has not been well understood so far. If the damping of beam instabilities is caused by the betatron tune spread (Landau damping) due to the nonlinearity of the space-charge force, one may naively think that a stronger space-charge force will be more effective in damping of beam instabilities. However, many simulation results show the contrary. This inversion may suggest that the damping phenomenon of beam instabilities in a weak

Published by the American Physical Society under the terms of the Creative Commons Attribution 3.0 License. Further distribution of this work must maintain attribution to the author(s) and the published article's title, journal citation, and DOI. space-charge region may come from a different mechanism (some possible explanations are proposed in Refs. [10,11]).

The two particle model has been applied to illustrate the mechanism of the strong head-tail instability (or the transverse mode-coupling instability [12]) in a very simple but insightful way (Ref. [13]: Chao, page 179). This two particle model can provide a superb framework for study of the space-charge force on beam instabilities just by adding new space-charge terms on top of the existing wake potential ones. The crucial points in this new model are that the resulting equations of motion need to be analytically solvable and the final form of solutions should be a continuous expansion from the one-dimensional (the wake strength only) case to the two-dimensional (the wake strength and the space-charge strength) case.

The purpose of the present simplified model is not to explain every effect of space-charge force on beam instabilities with numerical precision, but to suggest a simple picture of some of the essence of the physics of this complicated subject. We hope that it will provide a good starting point for readers to join this effort with their own models or improved versions of the present model so that the model becomes more physically accurate.

We briefly summarize Chao's no space-charge model in Sec. II to review the premise and the solution techniques of the original two particle model, and derive some useful formulas for later use. In Secs. III and IV, we solve the 
equations of motion for the weak and strong space-charge cases, respectively. The procedure to identify unstable regions and to compute the growth rate is summarized in Sec. V. Contour plots of the growth rate are presented in flat and three-dimensional ways. The paper is concluded with its findings in Sec. VI.

\section{NO SPACE-CHARGE CASE}

Let us first review the premise and treatment of Chao's original two particle model by closely following his textbook. We assume that a beam is made of two macroparticles, each with charge of $\mathrm{Ne} / 2$ and each executing smooth synchrotron and betatron oscillations. We assume that their synchrotron oscillations have equal amplitude, but opposite phases. As for the betatron oscillations, we make no such assumption for the amplitude and the phase. Throughout this paper, we only deal with the zero chromaticity case.

In what follows, we use $s$, the distance along the circumference, as an independent variable of motion. During the first half of the synchrotron oscillation period, $\mathrm{T}_{s}=2 \pi / \omega_{s}$, particle 2 leads particle 1 on the synchrotron phase space, and only the trailing particle (particle 1 in the present case) receives transverse kicks from wakefields created by the leading particle (particle 2 in the present case), that is a function of the transverse displacement of the leading particle. For simplicity, we assume that the wake potential is a constant $W_{0}$, independent of the distance between the two particles. The property of the wake potential requires that $W_{0}>0$. The equations of motion for the two particles are

$$
\begin{gathered}
\mathrm{y}_{1}^{\prime \prime}+\left(\frac{\omega_{\beta}}{c}\right)^{2} y_{1}=\frac{N r_{0} W_{0}}{2 \gamma C} y_{2}, \\
\mathrm{y}_{2}^{\prime \prime}+\left(\frac{\omega_{\beta}}{c}\right)^{2} y_{2}=0,
\end{gathered}
$$

where $\mathrm{y}^{\prime}=\frac{d y}{d s}, \omega_{\beta}$ is the betatron angular frequency, $c$ is the speed of light, $\gamma$ is the Lorenz factor, $C$ is the circumference of the machine, and $r_{0}$ is the classical radius of the particle. Similarly during the second half period of the synchrotron oscillation, we have the same equations with indices 1 and 2 exchanged.

The solution for $y_{2}$ is simply a free betatron oscillation:

$$
\tilde{\mathrm{y}}_{2}(s)=\tilde{\mathrm{y}}_{2}(0) e^{-i \omega_{\beta} s / c},
$$

where

$$
\tilde{\mathrm{y}}_{2}=y_{2}+i \frac{c}{\omega_{\beta}} y_{2}^{\prime}
$$

The solution for $y_{1}$ is simplified when the betatron frequency is much larger than the synchrotron one, $\omega_{\beta} \gg \omega_{s}$, which is mostly the case. It is then approximately given by

$$
\tilde{\mathrm{y}}_{1}(s)=\tilde{\mathrm{y}}_{1}(0) e^{-i \omega_{\beta} s / c}+i \Upsilon \tilde{\mathrm{y}}_{2}(0) e^{-i \omega_{\beta} s / c},
$$

where we have defined a positive dimensionless parameter for the wake potential strength

$$
\Upsilon=\frac{\pi N r_{0} W_{0} c^{2}}{4 \gamma C \omega_{\beta} \omega_{s}}
$$

We can write the solutions for the equations of motion during the period $0<\frac{s}{c}<T_{s} / 2$ in a matrix form as

$$
\left[\begin{array}{l}
\tilde{\mathrm{y}}_{1} \\
\tilde{\mathrm{y}}_{2}
\end{array}\right]_{s=c T_{s} / 2}=e^{-i \omega_{\beta} T_{s} / 2}\left[\begin{array}{cc}
1 & i \Upsilon \\
0 & 1
\end{array}\right]\left[\begin{array}{l}
\tilde{\mathrm{y}}_{1} \\
\tilde{\mathrm{y}}_{2}
\end{array}\right]_{s=0} .
$$

The transfer matrix during the second half of the synchrotron oscillation period, $T_{s} / 2<\frac{s}{c}<T_{s}$, is obtained by exchanging the indices 1 and 2 in the above treatment. The total transfer matrix for one full synchrotron oscillation period is then given by

$$
\begin{aligned}
{\left[\begin{array}{l}
\tilde{\mathrm{y}}_{1} \\
\tilde{\mathrm{y}}_{2}
\end{array}\right]_{s=c T_{s}} } & =e^{-i \omega_{\beta} T_{s}}\left[\begin{array}{cc}
1 & 0 \\
i \Upsilon & 1
\end{array}\right]\left[\begin{array}{cc}
1 & i \Upsilon \\
0 & 1
\end{array}\right]\left[\begin{array}{l}
\tilde{\mathrm{y}}_{1} \\
\tilde{\mathrm{y}}_{2}
\end{array}\right]_{s=0} \\
& =e^{-i \omega_{\beta} T_{s}}\left[\begin{array}{cc}
1 & i \Upsilon \\
i \Upsilon & 1-\Upsilon^{2}
\end{array}\right]\left[\begin{array}{l}
\tilde{\mathrm{y}}_{1} \\
\tilde{\mathrm{y}}_{2}
\end{array}\right]_{s=0} .
\end{aligned}
$$

Let us find eigenvalues $\lambda$ of this matrix by equating it as

$$
\begin{gathered}
e^{-i \omega_{\beta} T_{s}}\left[\begin{array}{cc}
1 & i \Upsilon \\
i \Upsilon & 1-\Upsilon^{2}
\end{array}\right]\left[\begin{array}{l}
\tilde{y}_{1} \\
\tilde{y}_{2}
\end{array}\right]_{s=0} \\
=e^{-i \omega_{\beta} T_{s}} \cdot \lambda\left[\begin{array}{ll}
1 & 0 \\
0 & 1
\end{array}\right]\left[\begin{array}{l}
\tilde{y}_{1} \\
\tilde{y}_{2}
\end{array}\right]_{s=0} .
\end{gathered}
$$

The two eigenvalues are found to be

$$
\lambda=\left\{\begin{array}{ll}
1-\frac{\Upsilon^{2}}{2} \pm \sqrt{\frac{\Upsilon^{2}}{2} \cdot\left(\frac{\Upsilon^{2}}{2}-2\right)} & \text { if } \Upsilon^{2} \geq 4 \\
1-\frac{\Upsilon^{2}}{2} \pm i \sqrt{\frac{\Upsilon^{2}}{2} \cdot\left(2-\frac{\Upsilon^{2}}{2}\right)} & \text { if } \Upsilon^{2} \leq 4
\end{array} .\right.
$$

Let us check the stability of the system for the two cases. If $\Upsilon^{2} \leq 4$, the square of the absolute value of $\lambda$ becomes

$$
|\lambda|^{2}=\left(1-\frac{\Upsilon^{2}}{2}\right)^{2}+\frac{\Upsilon^{2}}{2} \cdot\left(2-\frac{\Upsilon^{2}}{2}\right)=1 .
$$

Namely, the system is stable. If $\Upsilon^{2} \geq 4$, one of the solutions,

$$
\lambda=1-\frac{\Upsilon^{2}}{2}-\sqrt{\frac{\Upsilon^{2}}{2} \cdot\left(\frac{\Upsilon^{2}}{2}-2\right)} \leq-1
$$


with its amplitude larger than one, is unstable. At the threshold value of $\Upsilon^{2}=4$, the eigenvalue $\lambda$ becomes exactly minus one $(\lambda=-1)$. It can be expressed in the complex phase space as

$$
\lambda=e^{ \pm i \pi} .
$$

It indicates that during the full synchrotron oscillation period, the coherent betatron frequency shift, $\Delta \omega_{\beta}$, generates a betatron phase advance by $\pm \pi$. In other words,

$$
\left(\omega_{\beta}+\Delta \omega_{\beta}\right) \cdot T_{s}=\omega_{\beta} \cdot T_{s} \pm \pi .
$$

It shows that the strong head-tail instability occurs by the mode coupling between the two solutions when the difference of their betatron phase advances over one synchrotron period becomes exactly $2 \pi$. This mode coupling condition is different from the usual one that requires that the two modes share the same tune. This is because the present two particle model is dealing with the betatron phase advance over one synchrotron oscillation period, instead of a continuous interaction process between the two modes throughout the synchrotron oscillation.

The growth rate $g$, when $\Upsilon^{2} \geq 4$, is obtained by equating

$$
|\lambda|=e^{g T_{s}}=\sqrt{\frac{\Upsilon^{2}}{2} \cdot\left(\frac{\Upsilon^{2}}{2}-2\right)}+\frac{\Upsilon^{2}}{2}-1
$$

The formula for the growth rate is thus given by

$$
g=\frac{1}{T_{s}} \log \left\{\sqrt{\frac{\Upsilon^{2}}{2} \cdot\left(\frac{\Upsilon^{2}}{2}-2\right)}+\frac{\Upsilon^{2}}{2}-1\right\} .
$$

\section{WEAK SPACE-CHARGE CASE}

Now, let us introduce the space-charge force into the two particle model. Here, we make the following two approximations: (i) The space-charge force is linear in the relative distance between the two particles (the linear model). (ii) The two particles interact continuously and coherently with a space-charge force in the transverse plane (the continuous interaction model).

At a low energy (the Lorenz $\gamma \sim 1$ ) where the spacecharge force is significant, the space-charge fields spread out angularly with a large spread on the order of $1 / \gamma$, unlike a high-energy electron beam where the space-charge fields are Lorenz contracted into a thin disk. In this sense, two particles behave like rods, not point charges, each with a half bunch length of the total beam.

Under this linearized continuous interaction model, we have additional space-charge terms in the equations of motion for the period $0<\frac{\mathrm{s}}{\mathrm{c}}<T_{s} / 2$ as

$$
\begin{gathered}
\mathrm{y}_{1}^{\prime \prime}+\left(\frac{\omega_{\beta}}{c}\right)^{2} y_{1}=K\left(y_{1}-y_{2}\right)+W y_{2}, \\
\mathrm{y}_{2}^{\prime \prime}+\left(\frac{\omega_{\beta}}{c}\right)^{2} y_{2}=K\left(y_{2}-y_{1}\right) .
\end{gathered}
$$

Here, we define $\mathrm{W}$ as

$$
W=\frac{N r_{0} W_{0}}{2 \gamma C}
$$

and $\mathrm{K}$ denotes the space-charge kick strength:

$$
K=\frac{N r_{0}}{a^{2} \beta^{2} \gamma^{3} C},
$$

where $a$ is the transverse beam size and $\beta$ is the Lorenz $\beta$. The properties of the wake potential and the space-charge force require that both $\mathrm{W}$ and $\mathrm{K}$ are always positive:

$$
\mathrm{W}, \mathrm{K} \geq 0
$$

The ratio $\mathrm{r}=\mathrm{K} / \mathrm{W}$ can be expressed in a more familiar way, using $\Upsilon$ [defined by Eq. (6)] and the space-charge tune shift parameter $\Delta \nu_{s c}$ (normalized by the synchrotron tune $\left.\nu_{s}\right)$, as

$$
\mathrm{r}=\frac{\pi}{2 \Upsilon}\left(\frac{\Delta \nu_{s c}}{\nu_{s}}\right)
$$

For convenience, we define the space-charge tune shift parameter $\Delta \nu_{s c}$ as a modulus of the space-charge tune shift. The equations of motion can be written down in a slightly different way by moving the space-charge tune shift terms from the right-hand sides to the left-hand sides as

$$
\begin{gathered}
\mathrm{y}_{1}^{\prime \prime}+\left[\left(\frac{\omega_{\beta}}{c}\right)^{2}-K\right] y_{1}=(W-K) y_{2}, \\
\mathrm{y}_{2}^{\prime \prime}+\left[\left(\frac{\omega_{\beta}}{c}\right)^{2}-K\right] y_{2}=-K y_{1} .
\end{gathered}
$$

Hereafter, we assume that $\mathrm{W} \geq \mathrm{K}$, namely we deal with the weak space-charge case. Using the eigenvalue/eigenvector technique, the equations of motion can be readily solved (see Appendix A). The solutions can be written in a matrix form as

$$
\begin{aligned}
{\left[\begin{array}{c}
\tilde{y}_{1} \\
\tilde{y}_{2}
\end{array}\right]_{s=c T_{s} / 2}=} & e^{-i \theta_{1}}\left[\begin{array}{cc}
\cosh \theta_{2} & i \sqrt{\frac{W-K}{K}} \sinh \theta_{2} \\
-i \sqrt{\frac{K}{W-K}} \sinh \theta_{2} & \cosh \theta_{2}
\end{array}\right] \\
& \times\left[\begin{array}{c}
\tilde{y}_{1} \\
\tilde{y}_{2}
\end{array}\right]_{s=0},
\end{aligned}
$$

where 


$$
\theta_{1}=\left[\frac{\omega_{\beta}}{c}-\frac{1}{2} \frac{c}{\omega_{\beta}} K\right] \cdot \frac{c T_{s}}{2},
$$

and

$$
\theta_{2}=\frac{1}{2} \frac{c}{\omega_{\beta}} \sqrt{K(W-K)} \cdot \frac{c T_{s}}{2} .
$$

The transfer matrix during the other half period of the synchrotron oscillation $\left(T_{s} / 2<\frac{s}{c}<T_{s}\right)$ is given by

$$
\begin{aligned}
{\left[\begin{array}{l}
\tilde{y}_{1} \\
\tilde{y}_{2}
\end{array}\right]_{s=c T_{s}} } & =e^{-i \theta_{1}}\left[\begin{array}{cc}
\cosh \theta_{2} & -i \sqrt{\frac{K}{W-K}} \sinh \theta_{2} \\
i \sqrt{\frac{W-K}{K}} \sinh \theta_{2} & \cosh \theta_{2}
\end{array}\right] \\
& \times\left[\begin{array}{c}
\tilde{y}_{1} \\
\tilde{y}_{2}
\end{array}\right]_{s=c T_{s} / 2} .
\end{aligned}
$$

The total transfer matrix $\boldsymbol{T}$ for one full synchrotron oscillation period is then given by

$$
\begin{aligned}
\boldsymbol{T} & =e^{-2 i \theta_{1}}\left[\begin{array}{cc}
\cosh \theta_{2} & -i \sqrt{\frac{K}{W-K}} \sinh \theta_{2} \\
i \sqrt{\frac{W-K}{K}} \sinh \theta_{2} & \cosh \theta_{2}
\end{array}\right]\left[\begin{array}{cc}
\cosh \theta_{2} & i \sqrt{\frac{W-K}{K}} \sinh \theta_{2} \\
-i \sqrt{\frac{K}{W-K}} \sinh \theta_{2} & \cosh \theta_{2}
\end{array}\right] \\
& =e^{-2 i \theta_{1}}\left[\begin{array}{cc}
\cosh ^{2} \theta_{2}-\frac{K}{W-K} \sinh ^{2} \theta_{2} & i\left(\sqrt{\frac{W-K}{K}}-\sqrt{\frac{K}{W-K}}\right) \cosh \theta_{2} \sinh \theta_{2} \\
i\left(\sqrt{\frac{W-K}{K}}-\sqrt{\frac{K}{W-K}}\right) \cosh \theta_{2} \sinh \theta_{2} & \cosh ^{2} \theta_{2}-\frac{W-K}{K} \sinh ^{2} \theta_{2}
\end{array}\right] .
\end{aligned}
$$

The eigenvalues $\lambda$ of the total matrix $\boldsymbol{T}$ can be obtained by equating

$$
\boldsymbol{T}=e^{-2 i \theta_{1}} \lambda \boldsymbol{I}
$$

where $\boldsymbol{I}$ is the unit matrix. After some mathematical manipulations and using the relationship

$$
\cosh ^{2} \theta_{2}=1+\sinh ^{2} \theta_{2},
$$

we can write down the eigenvalues as

$$
\lambda=\left\{\begin{array}{ll}
1-\frac{\Gamma^{2}}{2} \pm \sqrt{\frac{\Gamma^{2}}{2} \cdot\left(\frac{\Gamma^{2}}{2}-2\right)} & \text { if } \Gamma^{2} \geq 4 \\
1-\frac{\Gamma^{2}}{2} \pm i \sqrt{\frac{\Gamma^{2}}{2} \cdot\left(2-\frac{\Gamma^{2}}{2}\right)} & \text { if } \Gamma^{2} \leq 4
\end{array} .\right.
$$

Here we have defined $\Gamma$ as

$$
\frac{\Gamma^{2}}{2}=(H-1) \sinh ^{2} \theta_{2} \geq 0,
$$

where

$$
\mathrm{H}=\frac{1}{2}\left(\frac{\mathrm{K}}{W-K}+\frac{\mathrm{W}-\mathrm{K}}{K}\right)
$$

and we have used that fact that $\mathrm{H}-1$ is always positive:

$$
\mathrm{H}-1=\frac{(W-2 K)^{2}}{2 K(W-K)} \geq 0 .
$$

Note the similar structure of the eigenvalues, Eq. (32), with those for no space-charge solutions, Eq. (10), where $\Gamma$ is just replaced by $\Upsilon$. We can make the exactly same argument on the stability of the system as in Sec. II for no space-charge case and conclude that the stability of the system requires that

$$
\Gamma^{2} \leq 4
$$

The growth rate $g$ for $\Gamma^{2} \geq 4$ is given by

$$
g=\frac{1}{T_{s}} \log \left\{\sqrt{\frac{\Gamma^{2}}{2} \cdot\left(\frac{\Gamma^{2}}{2}-2\right)}+\frac{\Gamma^{2}}{2}-1\right\} .
$$

The two eigenvalues for $\Gamma^{2} \leq 4$ can be expressed by the two vectors (green and red arrows) on the complex phase plane as illustrated in the left part of Fig. 1. The circle has a radius of one. The angle between each vector and the positive real axis is the phase advances of the corresponding eigenmode after one synchrotron oscillation period. At $\Gamma^{2}=4$, the difference of the betatron phase advances of the two eigenmodes over one synchrotron period becomes exactly $2 \pi$ and they line up on the negative real axis (the middle schematic of Fig. 1). That signals the onset of transverse mode coupling. When $\Gamma^{2} \geq 4$, one vector becomes longer than one (unstable mode), while the other vector becomes shorter than one (stable mode) as shown in the right part of Fig. 1.

The stability condition $\Gamma^{2} \leq 4$ can be expressed in a more convenient way using $\Upsilon$ defined by Eq. (6) and the ratio $\mathrm{r}=\mathrm{K} / \mathrm{W}$ between $\mathrm{K}$ and $\mathrm{W}$ (see Appendix $\mathrm{B}$ ) as

$$
\tanh ^{2}\left(\frac{\Upsilon}{2} \mathrm{y}\right) \leq y^{2}
$$

where $\mathrm{y}$ is defined by 


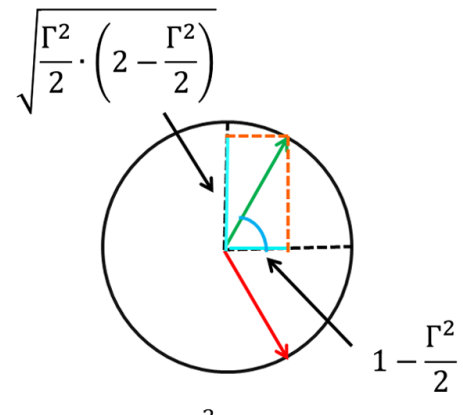

$$
\Gamma^{2} \leq 4
$$

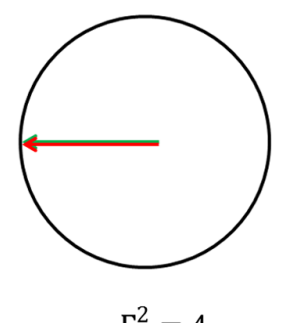

$\Gamma^{2}=4$

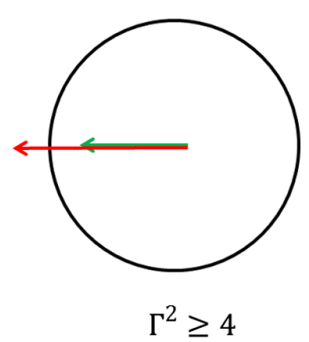

FIG. 1. Schematics of the eigenvalues on the complex phase plane: left for $\Gamma^{2} \leq 4$, middle for $\Gamma^{2}=4$, and right for $\Gamma^{2} \geq 4$.

$$
\mathrm{y}=2 \sqrt{r(1-r)}
$$

and

$$
\mathrm{r}=\frac{K}{W}
$$

Using the two parameters $\Upsilon$ and $y$, the $\Gamma^{2} / 2$ can be expressed as

$$
\frac{\Gamma^{2}}{2}=2 \cdot \frac{1-y^{2}}{y^{2}} \cdot \frac{\tanh ^{2}\left(\frac{\Upsilon}{2} y\right)}{1-\tanh ^{2}\left(\frac{\Upsilon}{2} y\right)} .
$$

As stated before, the ratio $\mathrm{r}=\mathrm{K} / \mathrm{W}$ can be expressed as

$$
\mathrm{r}=\frac{\pi}{2 \Upsilon}\left(\frac{\Delta \nu_{s c}}{\nu_{s}}\right)
$$

The stability criterion and the growth rate are now functions of the two dimensionless parameters: $\Upsilon$ and $\Delta \nu_{s c} / \nu_{s}$. The threshold value of $\Upsilon$ as a function of the ratio $r$ up to $r=1$ is plotted in Fig. 2. Unstable regions are shown shaded.

Without the space-charge force $(r=0)$, the threshold value of $\Upsilon$ for mode-coupling instability is 2 . As can be seen in Eqs. (23) and (24), the space-charge force rotates the betatron phase on the opposite direction to that for the wakefield. When a small amount of the space-charge force is introduced, a larger value of $\Upsilon$ is required to compensate the counterrotating contributions of the space-charge force so that the two eigenmode vectors in Fig. 1 will line up on the negative real axis and couple. That is why the stability threshold is going up at small $r$ regions in Fig. 2. When the space-charge force is increased to $r=1$, the phase advances by the space-charge force become exactly $\pm 2 \pi$ (namely, the two vectors rotate by $\pm 2 \pi$ to line up on the positive real axis), the threshold value of $\Upsilon$ for the mode-coupling instability becomes 2 again. The white region in Fig. 2 between $r=0$ and 1 and above $\Upsilon=2$ is a passband created by decoupling of the modes by the space-charge force.

\section{STRONG SPACE-CHARGE CASE}

If $\mathrm{K} \geq \mathrm{W}$ in the equations of motion for the half synchrotron oscillation period, $0<\frac{\mathrm{s}}{\mathrm{c}}<T_{s} / 2$, the solutions of Eqs. (23) and (24) become (see Appendix C)

$$
\left[\begin{array}{l}
\tilde{y}_{1} \\
\tilde{y}_{2}
\end{array}\right]_{s=\frac{c T_{s}}{2}}=e^{-i \theta_{1}}\left[\begin{array}{cc}
\cos \theta_{2} & i \sqrt{\frac{K-W}{K}} \sin \theta_{2} \\
i \sqrt{\frac{K}{K-W}} \sin \theta_{2} & \cos \theta_{2}
\end{array}\right]\left[\begin{array}{l}
\tilde{y}_{1} \\
\tilde{y}_{2}
\end{array}\right]_{s=0},
$$

where we define

$$
\theta_{2}=\frac{1}{2} \frac{c}{\omega_{\beta}} \sqrt{K(K-W)} \cdot \frac{c T_{s}}{2} .
$$

The transfer matrix during the other half period of the synchrotron oscillation $\left(T_{s} / 2<\frac{\mathrm{s}}{\mathrm{c}}<T_{s}\right)$ is given by

$$
\begin{aligned}
{\left[\begin{array}{c}
\tilde{y}_{1} \\
\tilde{y}_{2}
\end{array}\right]_{s=c T_{s}}=} & e^{-i \theta_{1}}\left[\begin{array}{cc}
\cos \theta_{2} & i \sqrt{\frac{K}{K-W}} \sin \theta_{2} \\
i \sqrt{\frac{K-W}{K}} \sin \theta_{2} & \cos \theta_{2}
\end{array}\right] \\
& \times\left[\begin{array}{c}
\tilde{y}_{1} \\
\tilde{y}_{2}
\end{array}\right]_{s=c T_{s} / 2} .
\end{aligned}
$$

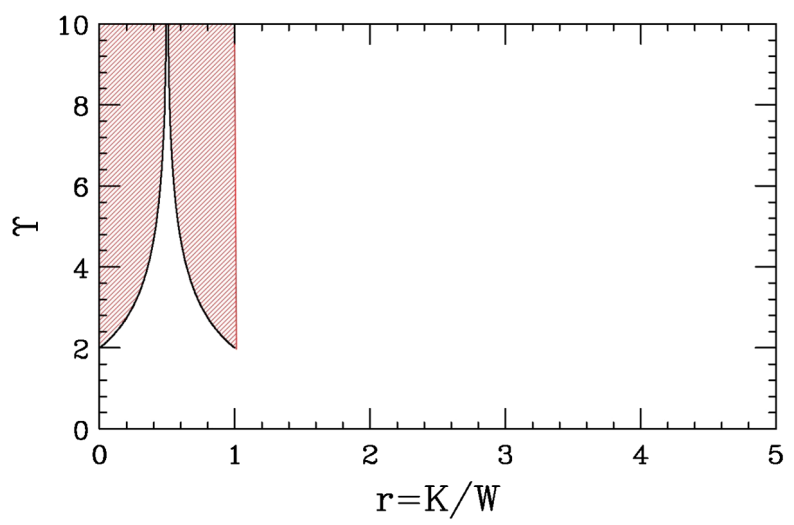

FIG. 2. The stability diagram for the weak space-charge case $(\mathrm{r}=\mathrm{K} / \mathrm{W} \leq 1)$. Unstable regions are shown shaded. 
The total transfer matrix $\boldsymbol{T}$ for one full synchrotron oscillation period is given by

$$
\boldsymbol{T}=e^{-2 i \theta_{1}}\left[\begin{array}{cc}
\cos ^{2} \theta_{2}-\frac{K}{K-W} \sin ^{2} \theta_{2} & i\left(\sqrt{\frac{K}{K-W}}+\sqrt{\frac{K-W}{K}}\right) \cos \theta_{2} \sin \theta_{2} \\
i\left(\sqrt{\frac{K}{K-W}}+\sqrt{\frac{K-W}{K}}\right) \cos \theta_{2} \sin \theta_{2} & \cos ^{2} \theta_{2}-\frac{K-W}{K} \sin ^{2} \theta_{2}
\end{array}\right] .
$$

The eigenvalues $\lambda$ of the total matrix $\boldsymbol{T}$ can be obtained by equating

$$
\boldsymbol{T}=e^{-2 i \theta_{1}} \lambda \boldsymbol{I} .
$$

After some mathematical manipulations and using the relationship

$$
\cos ^{2} \theta_{2}+\sin ^{2} \theta_{2}=1,
$$

we finally have the eigenvalues as

$$
\lambda=\left\{\begin{array}{ll}
1-\frac{\Gamma^{2}}{2} \pm \sqrt{\frac{\Gamma^{2}}{2} \cdot\left(\frac{\Gamma^{2}}{2}-2\right)} & \text { if } \Gamma^{2} \geq 4 \\
1-\frac{\Gamma^{2}}{2} \pm i \sqrt{\frac{\Gamma^{2}}{2} \cdot\left(2-\frac{\Gamma^{2}}{2}\right)} & \text { if } \Gamma^{2} \leq 4
\end{array} .\right.
$$

Here, we define $\Gamma$ as

$$
\frac{\Gamma^{2}}{2}=(H+1) \sin ^{2} \theta_{2} \geq 0,
$$

where we define $\mathrm{H}$ as

$$
\mathrm{H}=\frac{1}{2}\left(\frac{\mathrm{K}}{K-W}+\frac{\mathrm{K}-\mathrm{W}}{K}\right)
$$

and we use the fact that $\mathrm{H}+1$ is always positive:

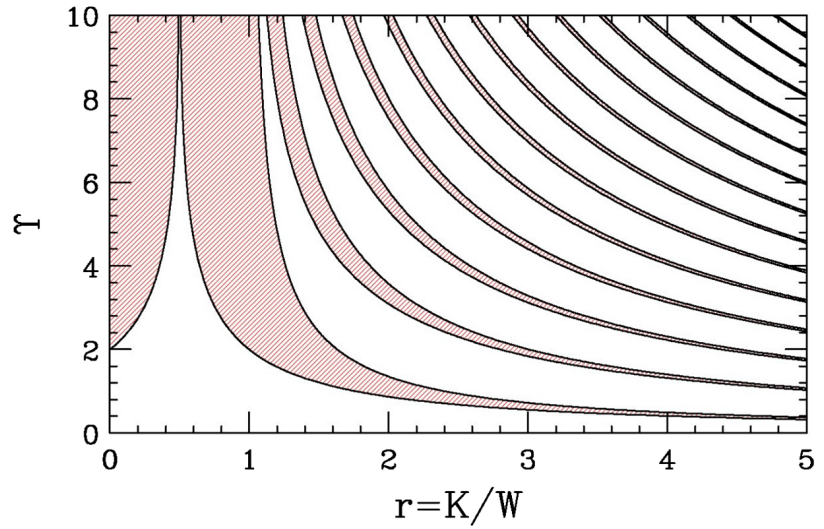

FIG. 3. The stability diagram for the strong space-charge case $(\mathrm{r}=\mathrm{K} / \mathrm{W} \geq 1)$. The stability diagram for the weak space-charge case $(r=K / W \leq 1)$ is also plotted for completion. Unstable regions are shown shaded.

$$
\mathrm{H}+1=\frac{(W-2 K)^{2}}{2 K(K-W)} \geq 0 .
$$

For the stability of the system, we can make the exactly same argument as before. The system is stable if

$$
\Gamma^{2} \leq 4
$$

The growth rate $g$ in unstable regions $\Gamma^{2} \geq 4$ is given by

$$
g=\frac{1}{T_{s}} \log \left\{\sqrt{\frac{\Gamma^{2}}{2} \cdot\left(\frac{\Gamma^{2}}{2}-2\right)}+\frac{\Gamma^{2}}{2}-1\right\} .
$$

The stability condition $\Gamma^{2} \leq 4$ can be expressed using $\Upsilon$ and the ratio $\mathrm{r}=\mathrm{K} / \mathrm{W}$ (see Appendix D) as

$$
\tan ^{2}\left(\frac{\Upsilon}{2} \mathrm{y}\right) \leq \mathrm{y}^{2},
$$

where $y$ is defined by

$$
\mathrm{y}=2 \sqrt{r(r-1)} .
$$

Using the two parameters $\Upsilon$ and $y$, the parameter $\Gamma^{2} / 2$ can be expressed as

$$
\frac{\Gamma^{2}}{2}=2 \cdot \frac{1+y^{2}}{y^{2}} \cdot \frac{\tan ^{2}\left(\frac{\Upsilon}{2} \mathrm{y}\right)}{1+\tan ^{2}\left(\frac{\Upsilon}{2} \mathrm{y}\right)} .
$$

The threshold value of $\Upsilon$ as a function of the ratio $r$ $(r \geq 1)$ is plotted in Fig. 3, together with the previous weak space-charge case $(\mathrm{r} \leq 1)$. Unstable regions are shown shaded.

\section{PROCEDURE TO IDENTIFY UNSTABLE REGIONS AND GROWTH RATE}

We now have the total solution for both the weak and strong space-charge regions. We can calculate the stability diagram using the following steps for given $\Upsilon$ and $\Delta \nu_{s c} / \nu_{s}$.

1. Calculate the ratio $\mathrm{r}$ :

$$
\mathrm{r}=\frac{K}{W}=\frac{\pi}{2 \Upsilon}\left(\frac{\Delta \nu_{s c}}{\nu_{s}}\right) .
$$


2. If $\mathrm{r} \leq 1$ (the weak space-charge case), calculate $\mathrm{y}$ as

$$
\mathrm{y}=2 \sqrt{r(1-r)} .
$$

2.1 If

$$
\tanh ^{2}\left(\frac{\Upsilon}{2} \mathrm{y}\right) \leq \mathrm{y}^{2}
$$

the beam is stable.

2.2 If

$$
\tanh ^{2}\left(\frac{\Upsilon}{2} \mathrm{y}\right) \geq \mathrm{y}^{2}
$$

the beam is unstable and the growth rate is given by

$$
g=\frac{1}{T_{s}} \log \left\{\sqrt{\frac{\Gamma^{2}}{2} \cdot\left(\frac{\Gamma^{2}}{2}-2\right)}+\frac{\Gamma^{2}}{2}-1\right\},
$$

where

$$
\frac{\Gamma^{2}}{2}=2 \cdot \frac{1-y^{2}}{y^{2}} \cdot \frac{\tanh ^{2}\left(\frac{\Upsilon}{2} \mathrm{y}\right)}{1-\tanh ^{2}\left(\frac{\Upsilon}{2} \mathrm{y}\right)} .
$$

3. If $r \geq 1$ (the strong space-charge case), calculate $y$ as

$$
\mathrm{y}=2 \sqrt{r(r-1)} .
$$

3.1 If

$$
\tan ^{2}\left(\frac{\Upsilon}{2} \mathrm{y}\right) \leq \mathrm{y}^{2}
$$

the beam is stable.

3.2 If

$$
\tan ^{2}\left(\frac{\Upsilon}{2} \mathrm{y}\right) \geq \mathrm{y}^{2}
$$

the beam is unstable and the growth rate is given by

$$
g=\frac{1}{T_{s}} \log \left\{\sqrt{\frac{\Gamma^{2}}{2} \cdot\left(\frac{\Gamma^{2}}{2}-2\right)}+\frac{\Gamma^{2}}{2}-1\right\},
$$

where

$$
\frac{\Gamma^{2}}{2}=2 \cdot \frac{1+y^{2}}{y^{2}} \cdot \frac{\tan ^{2}\left(\frac{\Upsilon}{2} \mathrm{y}\right)}{1+\tan ^{2}\left(\frac{\Upsilon}{2} \mathrm{y}\right)}
$$

We calculate the growth factor $\mathrm{g} \times T_{s}$ as a function of $\Upsilon$ and $\Delta \nu_{s c} / \nu_{s}$ and plot it in a flat contour plot in Fig. 4 and in a three-dimension contour plot in Fig. 5. The three parameters $\mathrm{g} \times \mathrm{T}_{s}, \Upsilon$ and $\Delta \nu_{s c} / \nu_{s}$ are all dimensionless parameters and these contour plots are universal.

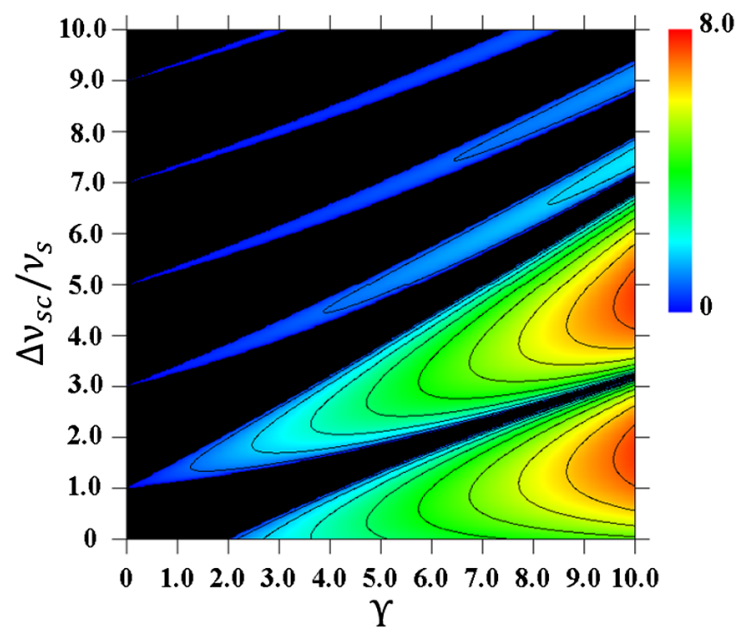

FIG. 4. Flat contour plot for the growth factor $\mathrm{g} \times \mathrm{T}_{s}$ as a function of $\Upsilon$ and $\frac{\Delta \nu_{s c}}{\nu_{s}}$.

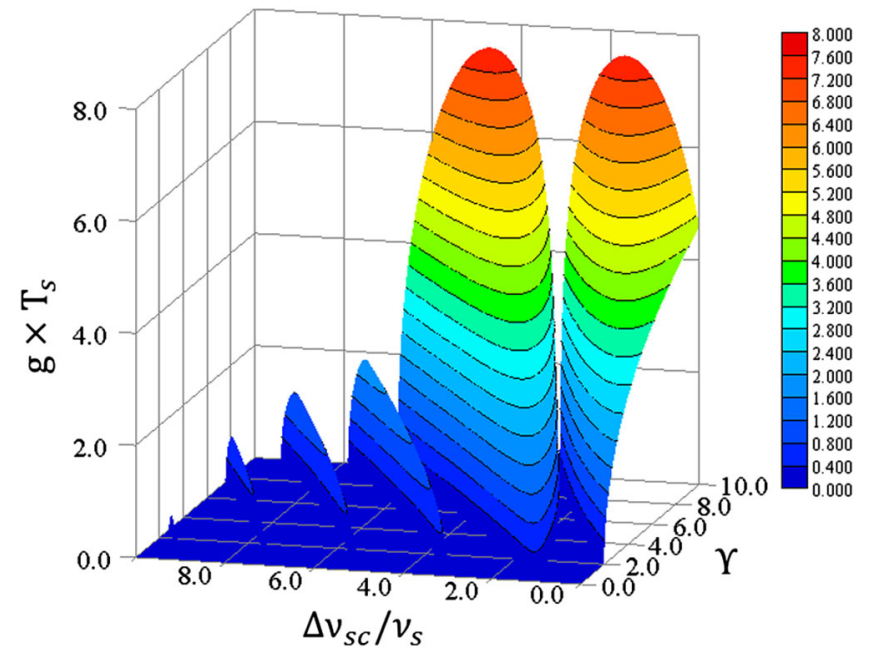

FIG. 5. Three-dimensional contour plot for the growth factor $\mathrm{g} \times \mathrm{T}_{s}$ as a function of $\Upsilon$ and $\frac{\Delta \nu_{s c}}{\nu_{s}}$.

\section{FINDINGS AND CONCLUSIONS}

Let us investigate how the space-charge force affects the strong head-tail instability. Take a case of $\Upsilon=4$ where the beam is unstable without the space-charge force $\left(\frac{\Delta \nu_{s c}}{\nu_{s}}=0\right)$. Figure 6 shows the growth factor $\mathrm{g} \times \mathrm{T}_{s}$ as a function of the space-charge tune shift parameter $\Delta \nu_{s c} / \nu_{s}$ (normalized by the synchrotron tune) at $\Upsilon=4$. If we gradually increase the space-charge force, the beam moves from the unstable region (the lowest stopband) to the stable region (the lowest passband). However, if we further increase the space-charge force, the beam enters another unstable region (the second lowest stopband). The maximum growth rate in this unstable region is comparable to that for no space-charge case. One may conclude that the space-charge force loses its damping effect when it is too strong. In fact, many theoretical and simulation studies show similar behaviors. If we further increase the space-charge force, the beam would be stable 


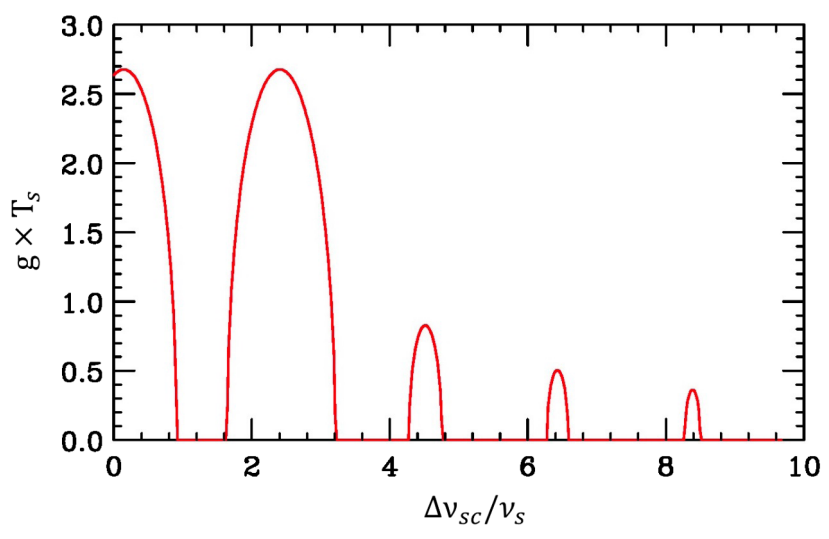

FIG. 6. The growth factor $\mathrm{g} \times \mathrm{T}_{s}$ as a function of the spacecharge tune shift parameter (normalized by the synchrotron tune) at $\Upsilon=4$.

again. However, it is not clear if we can achieve this state in reality or computer simulations since such strong spacecharge force may expand the beam size, with a result of reduction of the space-charge tune shift by itself.

One can see stopband structures in the stability diagrams Figs. 4 and 5. The lowest two stopbands are strongest (namely, very unstable), while other higher-order stopbands are considerably weaker. The appearance of the stopbands can be explained as follows. A mode-coupling instability takes place when the two eigenvalues line up on the negative real axis on the complex phase plane (see Fig. 1), in other words, when the difference of their betatron phase advances over one synchrotron period is an odd integer times $2 \pi$. When the wakefield is negligibly weak, the tune shift of one solution is close to $-\Delta \nu_{s c}$, while the other solution has almost no tune shift. The mode-coupling condition mentioned above corresponds to the case when $\Delta \nu_{s c}$ takes values around an odd integer times $\nu_{s}$. That is why the stopbands always start with $\Delta \nu_{s c} / \nu_{s}$ equal to odd integers at small $\Upsilon$ in Fig. 4. Pure space-charge oscillations are stable, but even slight inclusion of wakefield effects to them can make such oscillations unstable.

The present two particle model has no tune spread effect, since the space-charge force is linearized in the transverse position, and the two particles have identical betatron tunes. The damping of strong head-tail instabilities with a weak space-charge force is caused by decoupling of the modes due to additional tune shifts by the space-charge force, not the Landau damping due to the nonlinearity of the space-charge force. As the space-charge force is increased, tune shifts by the space-charge force conversely restore the mode coupling. But, a further increase of the space-charge force decuples the modes again. This mode coupling/decoupling behavior creates stopband structures as a function of the space-charge tune shift parameter and $\Upsilon$. This conclusion is consistent with the work by $\mathrm{Ng}$ and Burov [14], though their model includes many more modes. It is interesting to point out that in the present two particle model, there are only two modes,

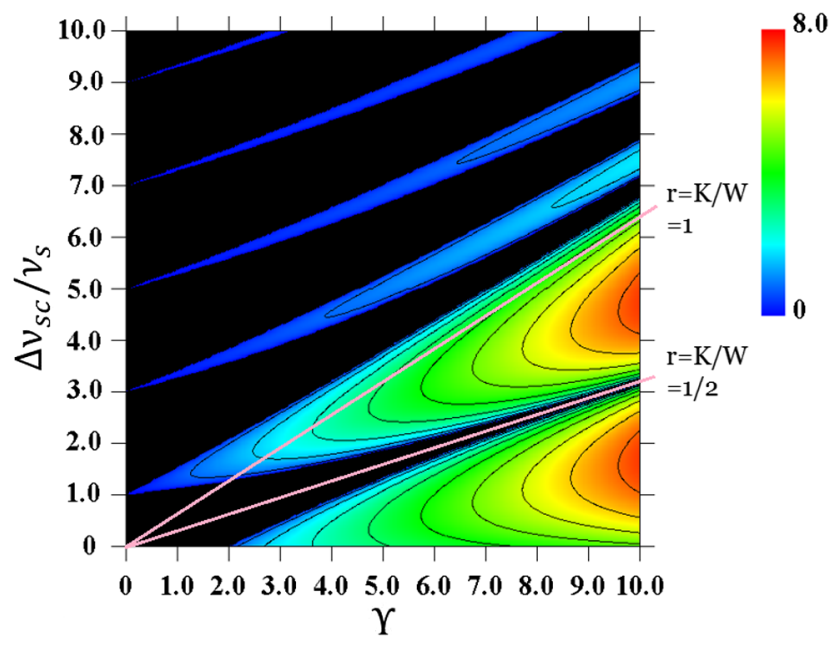

FIG. 7. Flat contour plot for the growth factor $\mathrm{g} \times \mathrm{T}_{s}$ as a function of $\Upsilon$ and $\frac{\Delta \nu_{s p}}{\nu_{s}}$. The cases $\mathrm{r}=\mathrm{K} / \mathrm{W}=1 / 2$ and $\mathrm{r}=$ $\mathrm{K} / \mathrm{W}=1$ are shown in red lines.

but they play many different modes as if in a more general analysis based on the mode expansion method [14]. Very roughly speaking, it appears that the one mode plays always the $\mathrm{m}=0$ mode, while the other mode plays negative odd integer modes $(\mathrm{m}=-1,-3,-5, \ldots)$ depending on the strength of the space-charge force.

For the sake of explanation, we draw two lines in the contour plot Fig. 7: one for $r=K / W=1 / 2$ and another for $\mathrm{r}=\mathrm{K} / \mathrm{W}=1$. The narrow passband region between the two lowest stopbands corresponds to the $\mathrm{r}=\mathrm{K} / \mathrm{W}=1 / 2$ case. In this case, the equations of motion become symmetric about $y_{1}$ and $y_{2}$, though the signs of the right-hand sides are opposite. When $\mathrm{K}$ is exactly equal to $\mathrm{W} / 2$, this system is absolutely stable regardless of the strength of $\mathrm{K}=\mathrm{W} / 2$. Another absolutely stable case is the $\mathrm{W}=0$ case, namely, no wakefield case. Then, the system becomes identical to that for two pendulums connected with a spring. This system is also stable regardless of the strength of the spring. In our case, the system starts to approach this extreme once the space-charge tune shift exceeds the $\mathrm{r}=\mathrm{K} / \mathrm{W}=1$ line. That may explain why the high-order stopbands are considerably weaker than the lowest two stopbands.

Although the present new two particle model is a simple expansion of the existing two particle model, it contains rich new physics. Further investigation of the present model and/or inclusion of more effects will help us to have a better understanding of effects of space-charge force on beam instabilities. We are planning to include the chromaticity effect to the present two particle model in the next report.

\section{ACKNOWLEDGMENTS}

This work was supported by U.S. Department of Energy Contracts No. DE-SC0012704 and DEAC02-76SF00515. 


\section{APPENDIX A: SOLUTIONS FOR EQUATIONS OF MOTION FOR WEAK SPACE-CHARGE FORCE $(\mathbf{W} \geq \mathbf{K})$}

Let us start with Eqs. (23) and (24):

$$
\begin{gathered}
\mathrm{y}_{1}^{\prime \prime}+\left[\left(\frac{\omega_{\beta}}{c}\right)^{2}-K\right] y_{1}=(W-K) y_{2}, \\
\mathrm{y}_{2}^{\prime \prime}+\left[\left(\frac{\omega_{\beta}}{c}\right)^{2}-K\right] y_{2}=-K y_{1} .
\end{gathered}
$$

Let us define

$$
\mathrm{A}^{2}=\left(\frac{\omega_{\beta}}{c}\right)^{2}-K
$$

Then, Eqs. (A1) and (A2) can be expressed in a matrix form as

$$
\left[\begin{array}{l}
\mathrm{y}_{1}^{\prime \prime} \\
\mathrm{y}_{2}^{\prime \prime}
\end{array}\right]=\left[\begin{array}{cc}
-A^{2} & W-K \\
-K & -A^{2}
\end{array}\right]\left[\begin{array}{l}
y_{1} \\
y_{2}
\end{array}\right]=\lambda\left[\begin{array}{l}
y_{1} \\
y_{2}
\end{array}\right]
$$

The eigenvalues $\lambda$ of this matrix are

$$
\lambda=-\mathrm{A}^{2} \pm i \sqrt{K(W-K)} .
$$

The eigenvectors have to satisfy

$$
\left[\left(\begin{array}{ll}
\lambda & 0 \\
0 & \lambda
\end{array}\right)-\left(\begin{array}{cc}
-A^{2} & W-K \\
-K & -A^{2}
\end{array}\right)\right]\left[\begin{array}{l}
x_{1} \\
x_{2}
\end{array}\right]=0
$$

The solutions are given by

$$
\left[\begin{array}{l}
x_{1} \\
x_{2}
\end{array}\right]=\left[\begin{array}{c}
\mp i \sqrt{K(W-K)} \\
K
\end{array}\right] .
$$

Let us define new coordinates:

$$
z_{1}=y_{1}+i \sqrt{\frac{W-K}{K}} y_{2}
$$

and

$$
z_{2}=y_{1}-i \sqrt{\frac{W-K}{K}} y_{2} .
$$

Then, the equations of motion can be diagonalized for $z_{1}$ and $z_{2}$ as

$$
\mathrm{z}_{1}^{\prime \prime}+A^{2} z_{1}=-i \sqrt{K(W-K)} z_{1}
$$

and

$$
\mathrm{z}_{2}^{\prime \prime}+A^{2} z_{2}=i \sqrt{K(W-K)} z_{2} .
$$

Let us define

$$
B_{ \pm}^{2}=A^{2} \pm i \sqrt{K(W-K)} .
$$

Then, solutions of Eqs. (A10) and (A11) are

$$
\tilde{\mathrm{z}}_{1}(s)=\tilde{\mathrm{z}}_{1}(0) e^{-i B_{+} s}
$$

and

$$
\tilde{\mathrm{z}}_{2}(s)=\tilde{\mathrm{z}}_{2}(0) e^{-i B_{-} s},
$$

where

$$
\tilde{\mathrm{z}}_{1,2}=z_{1,2}+i \frac{c}{\omega_{\beta}} z_{1,2}^{\prime}
$$

Solutions for

$$
\tilde{\mathrm{y}}_{1,2}=y_{1,2}+i \frac{c}{\omega_{\beta}} y_{1,2}^{\prime}
$$

are given by

$$
\begin{aligned}
\tilde{\mathrm{y}}_{1}(s)= & \frac{1}{2}\left(e^{-i B_{+} s}+e^{-i B_{-} s}\right) \tilde{\mathrm{y}}_{1}(0) \\
& +\frac{1}{2} \sqrt{\frac{W-K}{K}}\left(e^{-i B_{+} s}-e^{-i B_{-} s}\right) \tilde{\mathrm{y}}_{2}(0),
\end{aligned}
$$

and

$$
\begin{aligned}
\tilde{\mathrm{y}}_{2}(s)= & \frac{1}{i \sqrt{\frac{W-K}{K}}} \cdot \frac{1}{2}\left(e^{-i B_{+} s}-e^{-i B_{-} s}\right) \tilde{\mathrm{y}}_{1}(0) \\
& +\frac{1}{2}\left(e^{-i B_{+} s}+e^{-i B_{-} s}\right) \tilde{\mathrm{y}}_{2}(0) .
\end{aligned}
$$

Let us define

$$
\alpha_{+}=\frac{1}{2}\left(e^{-i B_{+} s}+e^{-i B_{-} s}\right)
$$

and

$$
\alpha_{-}=\frac{1}{2}\left(e^{-i B_{+} s}-e^{-i B_{-} s}\right) .
$$

The parameter $B_{ \pm}^{2}=A^{2} \pm i \sqrt{K(W-K)}$ can be approximated when $\mathrm{A} \gg \mathrm{W}, \mathrm{K}$ as

$$
\mathrm{B}_{ \pm} \cong A \pm \frac{i}{2 A} \sqrt{K(W-K)}
$$

where $A$ can be also approximated by

$$
\mathrm{A} \cong \frac{\omega_{\beta}}{c}-\frac{c}{2 \omega_{\beta}} K .
$$


Therefore,

$$
\mathrm{B}_{ \pm} \cong \frac{\omega_{\beta}}{c}-\frac{c}{2 \omega_{\beta}} K \pm \mathrm{i} \frac{c}{2 \omega_{\beta}} \sqrt{K(W-K)} .
$$

The error associated with the approximation (A23) is on the order of the square of the space-charge tune shift divided by the betatron tune [3], which is very small for any reasonable accelerator parameters.

The parameters $\alpha_{+}$and $\alpha_{-}$can be more explicitly written as

$\alpha_{+}=\exp \left[-i\left(\frac{\omega_{\beta}}{c}-\frac{c}{2 \omega_{\beta}} K\right) s\right] \cosh \left[\frac{c}{2 \omega_{\beta}} \sqrt{K(W-K)} s\right]$

and

$\alpha_{-}=\exp \left[-i\left(\frac{\omega_{\beta}}{c}-\frac{c}{2 \omega_{\beta}} K\right) s\right] \sinh \left[\frac{c}{2 \omega_{\beta}} \sqrt{K(W-K)} s\right]$.

The transfer matrix for the period $0<\frac{\mathrm{s}}{\mathrm{c}}<T_{s} / 2$ becomes

$$
\begin{aligned}
{\left[\begin{array}{c}
\tilde{y}_{1} \\
\tilde{y}_{2}
\end{array}\right]_{s=c T_{s} / 2}=} & e^{-i \theta_{1}}\left[\begin{array}{cc}
\cosh \theta_{2} & i \sqrt{\frac{W-K}{K}} \sinh \theta_{2} \\
-i \sqrt{\frac{K}{W-K}} \sinh \theta_{2} & \cosh \theta_{2}
\end{array}\right] \\
& \times\left[\begin{array}{c}
\tilde{y}_{1} \\
\tilde{y}_{2}
\end{array}\right]_{s=0},
\end{aligned}
$$

where

$$
\theta_{1}=\left[\frac{\omega_{\beta}}{c}-\frac{1}{2} \frac{c}{\omega_{\beta}} K\right] \cdot \frac{c T_{s}}{2},
$$

and

$$
\theta_{2}=\frac{1}{2} \frac{c}{\omega_{\beta}} \sqrt{K(W-K)} \cdot \frac{c T_{s}}{2} .
$$

The transfer matrix during the other half period of the synchrotron oscillation $\left(T_{s} / 2<\frac{\mathrm{s}}{\mathrm{c}}<T_{s}\right)$ is given by

$$
\begin{aligned}
{\left[\begin{array}{c}
\tilde{y}_{1} \\
\tilde{y}_{2}
\end{array}\right]_{s=c T_{s}}=} & e^{-i \theta_{1}}\left[\begin{array}{cc}
\cosh \theta_{2} & -i \sqrt{\frac{K}{W-K}} \sinh \theta_{2} \\
i \sqrt{\frac{W-K}{K}} \sinh \theta_{2} & \cosh \theta_{2}
\end{array}\right] \\
& \times\left[\begin{array}{c}
\tilde{y}_{1} \\
\tilde{y}_{2}
\end{array}\right]_{s=c T_{s} / 2} \cdot
\end{aligned}
$$

The total transfer matrix $\boldsymbol{T}$ for one full synchrotron oscillation period is then given by

$$
\begin{aligned}
\boldsymbol{T} & =e^{-2 i \theta_{1}}\left[\begin{array}{cc}
\cosh \theta_{2} & -i \sqrt{\frac{K}{W-K}} \sinh \theta_{2} \\
i \sqrt{\frac{W-K}{K}} \sinh \theta_{2} & \cosh \theta_{2}
\end{array}\right]\left[\begin{array}{cc}
\cosh \theta_{2} & i \sqrt{\frac{W-K}{K}} \sinh \theta_{2} \\
-i \sqrt{\frac{K}{W-K}} \sinh \theta_{2} & \cosh \theta_{2}
\end{array}\right] \\
= & e^{-2 i \theta_{1}}\left[\begin{array}{cc}
\cosh ^{2} \theta_{2}-\frac{K}{W-K} \sinh ^{2} \theta_{2} & i\left(\sqrt{\frac{W-K}{K}}-\sqrt{\frac{K}{W-K}}\right) \cosh \theta_{2} \sinh \theta_{2} \\
i\left(\sqrt{\frac{W-K}{K}}-\sqrt{\frac{K}{W-K}}\right) \cosh \theta_{2} \sinh \theta_{2} & \cosh ^{2} \theta_{2}-\frac{W-K}{K} \sinh ^{2} \theta_{2}
\end{array}\right] .
\end{aligned}
$$

The eigenvalues $\lambda$ of the total matrix $\boldsymbol{T}$ can be obtained by equating

$$
\boldsymbol{T}=e^{-2 i \theta_{1}} \lambda \boldsymbol{I},
$$

where $I$ is the unit matrix. After some mathematical manipulations and using the relationship

$$
\cosh ^{2} \theta_{2}=1+\sinh ^{2} \theta_{2},
$$

we can write down the eigenvalues as

$$
\lambda= \begin{cases}1-\frac{\Gamma^{2}}{2} \pm \sqrt{\frac{\Gamma^{2}}{2} \cdot\left(\frac{\Gamma^{2}}{2}-2\right)} & \text { if } \Gamma^{2} \geq 4 \\ 1-\frac{\Gamma^{2}}{2} \pm i \sqrt{\frac{\Gamma^{2}}{2} \cdot\left(2-\frac{\Gamma^{2}}{2}\right)} & \text { if } \Gamma^{2} \leq 4\end{cases}
$$

Here we have defined $\Gamma$ as

$$
\frac{\Gamma^{2}}{2}=(H-1) \sinh ^{2} \theta_{2} \geq 0,
$$

where 


$$
\mathrm{H}=\frac{1}{2}\left(\frac{\mathrm{K}}{W-K}+\frac{\mathrm{W}-\mathrm{K}}{K}\right)
$$

and we have used the fact that $\mathrm{H}-1$ is always positive:

$$
\mathrm{H}-1=\frac{(W-2 K)^{2}}{2 K(W-K)} \geq 0 .
$$

\section{APPENDIX B: STABILITY CONDITION FOR WEAK SPACE-CHARGE FORCE $(\mathrm{W} \geq \mathrm{K})$}

The stability of the system requires that

$$
\Gamma^{2} \leq 4
$$

This stability condition can be expressed more explicitly as

$$
\tanh ^{2} \theta_{2} \leq \frac{4 K(W-K)}{W^{2}} .
$$

Since

$$
\theta_{2}=\frac{1}{2} \frac{c}{\omega_{\beta}} \sqrt{K(W-K)} \cdot \frac{c T_{s}}{2}=\frac{y}{2} \Upsilon
$$

where

$$
\begin{gathered}
\Upsilon=\frac{1}{2} W \frac{c}{\omega_{\beta}} \frac{c T_{s}}{2}, \\
y=\frac{2 \sqrt{K(W-K)}}{W}=2 \sqrt{r(1-r)},
\end{gathered}
$$

and

$$
\mathrm{r}=\frac{K}{W},
$$

the stability condition can be written as

$$
\tanh ^{2}\left(\frac{\Upsilon}{2} y\right) \leq \mathrm{y}^{2}
$$

Using the two parameters $\Upsilon$ and $y$, the $\Gamma^{2} / 2$ can be expressed as

$$
\frac{\Gamma^{2}}{2}=2 \cdot \frac{1-y^{2}}{y^{2}} \cdot \frac{\tanh ^{2}\left(\frac{\Upsilon}{2} \mathrm{y}\right)}{1-\tanh ^{2}\left(\frac{\Upsilon}{2} \mathrm{y}\right)} .
$$

The ratio $\mathrm{r}=\mathrm{K} / \mathrm{W}$ can be expressed as

$$
\mathrm{r}=\frac{\pi}{2 \Upsilon}\left(\frac{\Delta \nu_{s c}}{\nu_{s}}\right)
$$

\section{APPENDIX C: SOLUTIONS FOR EQUATIONS OF MOTION FOR STRONG SPACE-CHARGE FORCE $(K \geq \mathbf{W})$}

In this region, the eigenvalues of the matrix

$$
\left[\begin{array}{l}
\mathrm{y}_{1}^{\prime \prime} \\
\mathrm{y}_{2}^{\prime \prime}
\end{array}\right]=\left[\begin{array}{cc}
-A^{2} & W-K \\
-K & -A^{2}
\end{array}\right]\left[\begin{array}{l}
y_{1} \\
y_{2}
\end{array}\right]=\lambda\left[\begin{array}{l}
y_{1} \\
y_{2}
\end{array}\right]
$$

are given by

$$
\lambda=-\mathrm{A}^{2} \pm \sqrt{K(K-W)} .
$$

The eigenvectors are given by

$$
\left[\begin{array}{l}
x_{1} \\
x_{2}
\end{array}\right]=\left[\begin{array}{c} 
\pm \sqrt{K(K-W)} \\
K
\end{array}\right] .
$$

Let us define new coordinates:

$$
z_{1}=y_{1}+\sqrt{\frac{K-W}{K}} y_{2},
$$

and

$$
z_{2}=y_{1}-\sqrt{\frac{K-W}{K}} y_{2} .
$$

Then, the equations of motion can be diagonalized for $z_{1}$ and $z_{2}$ as

$$
\mathrm{z}_{1}^{\prime \prime}+A^{2} z_{1}=-\sqrt{K(K-W)} z_{1}
$$

and

$$
\mathrm{z}_{2}^{\prime \prime}+A^{2} z_{2}=\sqrt{K(K-W)} z_{2} .
$$

Let us define

$$
B_{ \pm}^{2}=A^{2} \pm \sqrt{K(K-W)} .
$$

Then, the solutions of Eqs. (C6) and (C7) are

$$
\tilde{\mathrm{z}}_{1}(s)=\tilde{\mathrm{z}}_{1}(0) e^{-i B_{+} s}
$$

and

$$
\tilde{\mathrm{z}}_{2}(s)=\tilde{\mathrm{z}}_{2}(0) e^{-i B_{-} s},
$$

where

$$
\tilde{\mathrm{z}}_{1,2}=z_{1,2}+i \frac{c}{\omega_{\beta}} z_{1,2}^{\prime} .
$$

The solutions for

$$
\tilde{\mathrm{y}}_{1,2}=y_{1,2}+i \frac{c}{\omega_{\beta}} y_{1,2}^{\prime}
$$


are given by

$$
\begin{aligned}
\tilde{\mathrm{y}}_{1}(s)= & \frac{1}{2}\left(e^{-i B_{+} s}+e^{-i B_{-} s}\right) \tilde{\mathrm{y}}_{1}(0) \\
& +\frac{1}{2} \sqrt{\frac{K-W}{K}}\left(e^{-i B_{+} s}-e^{-i B_{-} s}\right) \tilde{\mathrm{y}}_{2}(0)
\end{aligned}
$$

and

$$
\begin{aligned}
\tilde{\mathrm{y}}_{2}(s)= & \sqrt{\frac{K}{K-W}} \cdot \frac{1}{2}\left(e^{-i B_{+} s}-e^{-i B_{-} s}\right) \tilde{\mathrm{y}}_{1}(0) \\
& +\frac{1}{2}\left(e^{-i B_{+} s}+e^{-i B_{-} s}\right) \tilde{\mathrm{y}}_{2}(0) .
\end{aligned}
$$

Let us define

$$
\alpha_{+}=\frac{1}{2}\left(e^{-i B_{+} s}+e^{-i B_{-} s}\right)
$$

and

$$
\alpha_{-}=\frac{1}{2}\left(e^{-i B_{+} s}-e^{-i B_{-} s}\right) .
$$

The parameters $\alpha_{+}$and $\alpha_{-}$can be more explicitly written as

$$
\alpha_{+}=\exp \left[-i\left(\frac{\omega_{\beta}}{c}-\frac{c}{2 \omega_{\beta}} K\right) s\right] \cos \left[\frac{c}{2 \omega_{\beta}} \sqrt{K(K-W)} s\right]
$$

and

$$
\boldsymbol{T}=e^{-2 i \theta_{1}}\left[\begin{array}{cc}
\cos ^{2} \theta_{2}-\frac{K}{K-W} \sin ^{2} \theta_{2} & i\left(\sqrt{\frac{K}{K-W}}+\sqrt{\frac{K-W}{K}}\right) \cos \theta_{2} \sin \theta_{2} \\
i\left(\sqrt{\frac{K}{K-W}}+\sqrt{\frac{K-W}{K}}\right) \cos \theta_{2} \sin \theta_{2} & \cos ^{2} \theta_{2}-\frac{K-W}{K} \sin ^{2} \theta_{2}
\end{array}\right] .
$$

The eigenvalues $\lambda$ of the total matrix $\boldsymbol{T}$ can be obtained by equating

$$
\boldsymbol{T}=e^{-2 i \theta_{1}} \lambda \boldsymbol{I}
$$

After some mathematical manipulations and using the relationship

$$
\cos ^{2} \theta_{2}+\sin ^{2} \theta_{2}=1,
$$

we finally have the eigenvalues as

$$
\alpha_{-}=i \exp \left[-i\left(\frac{\omega_{\beta}}{c}-\frac{c}{2 \omega_{\beta}} K\right) s\right] \sin \left[\frac{c}{2 \omega_{\beta}} \sqrt{K(K-W)} s\right] .
$$

For the half synchrotron oscillation period, $0<\frac{\mathrm{s}}{\mathrm{c}}<T_{s} / 2$, the transfer matrix becomes

$$
\begin{aligned}
{\left[\begin{array}{c}
\tilde{y}_{1} \\
\tilde{y}_{2}
\end{array}\right]_{s=\frac{c T_{s}}{2}}=} & e^{-i \theta_{1}}\left[\begin{array}{cc}
\cos \theta_{2} & i \sqrt{\frac{K-W}{K}} \sin \theta_{2} \\
i \sqrt{\frac{K}{K-W}} \sin \theta_{2} & \cos \theta_{2}
\end{array}\right] \\
& \times\left[\begin{array}{c}
\tilde{y}_{1} \\
\tilde{y}_{2}
\end{array}\right]_{s=0},
\end{aligned}
$$

where

$$
\theta_{2}=\frac{1}{2} \frac{c}{\omega_{\beta}} \sqrt{K(K-W)} \cdot \frac{c T_{s}}{2} .
$$

The transfer matrix during the other half period of the synchrotron oscillation $\left(T_{s} / 2<\frac{\mathrm{s}}{\mathrm{c}}<T_{s}\right)$ is given by

$$
\begin{aligned}
{\left[\begin{array}{c}
\tilde{y}_{1} \\
\tilde{y}_{2}
\end{array}\right]_{s=c T_{s}}=} & e^{-i \theta_{1}}\left[\begin{array}{cc}
\cos \theta_{2} & i \sqrt{\frac{K}{K-W}} \sin \theta_{2} \\
i \sqrt{\frac{K-W}{K}} \sin \theta_{2} & \cos \theta_{2}
\end{array}\right] \\
& \times\left[\begin{array}{c}
\tilde{y}_{1} \\
\tilde{y}_{2}
\end{array}\right]_{s=c T_{s} / 2} .
\end{aligned}
$$

The total transfer matrix $\boldsymbol{T}$ for one full synchrotron oscillation period is given by

$$
\lambda= \begin{cases}1-\frac{\Gamma^{2}}{2} \pm \sqrt{\frac{\Gamma^{2}}{2} \cdot\left(\frac{\Gamma^{2}}{2}-2\right)} & \text { if } \Gamma^{2} \geq 4 \\ 1-\frac{\Gamma^{2}}{2} \pm i \sqrt{\frac{\Gamma^{2}}{2} \cdot\left(2-\frac{\Gamma^{2}}{2}\right)} & \text { if } \Gamma^{2} \leq 4\end{cases}
$$

Here, we define $\Gamma$ as

$$
\frac{\Gamma^{2}}{2}=(H+1) \sin ^{2} \theta_{2} \geq 0,
$$

where $\mathrm{H}$ is defined as

$$
\mathrm{H}=\frac{1}{2}\left(\frac{\mathrm{K}}{K-W}+\frac{\mathrm{K}-\mathrm{W}}{K}\right)
$$


and we use the fact that $\mathrm{H}+1$ is always positive:

$$
\mathrm{H}+1=\frac{(W-2 K)^{2}}{2 K(K-W)} \geq 0 .
$$

The system is stable if

$$
\Gamma^{2} \leq 4
$$

The growth rate $g$ in unstable regions $\Gamma^{2} \geq 4$ is given by

$$
g=\frac{1}{T_{s}} \log \left\{\sqrt{\frac{\Gamma^{2}}{2} \cdot\left(\frac{\Gamma^{2}}{2}-2\right)}+\frac{\Gamma^{2}}{2}-1\right\} .
$$

\section{APPENDIX D: STABILITY CONDITION FOR STRONG SPACE-CHARGE FORCE $(K \geq \mathbf{W})$}

The stability of the system requires that

$$
\Gamma^{2} \leq 4
$$

This stability condition can be expressed more explicitly as

$$
\tan ^{2}\left(\frac{\Upsilon}{2} \mathrm{y}\right) \leq \mathrm{y}^{2}
$$

where $\mathrm{y}$ is defined as

$$
\mathrm{y}=\frac{2 \sqrt{K(K-W)}}{W}=2 \sqrt{r(r-1)},
$$

and

$$
\mathrm{r}=\frac{K}{W}
$$

Using the two parameters $\Upsilon$ and $y$, the $\Gamma^{2} / 2$ can be expressed as

$$
\frac{\Gamma^{2}}{2}=2 \cdot \frac{1+y^{2}}{y^{2}} \cdot \frac{\tan ^{2}\left(\frac{\Upsilon}{2} \mathrm{y}\right)}{1+\tan ^{2}\left(\frac{\Upsilon}{2} \mathrm{y}\right)} .
$$

The ratio $\mathrm{r}=\mathrm{K} / \mathrm{W}$ can be expressed as

$$
\mathrm{r}=\frac{\pi}{2 \Upsilon}\left(\frac{\Delta \nu_{s c}}{\nu_{s}}\right) .
$$

[1] M. Blaskiewicz, Fast head-tail instability with space charge, Phys. Rev. ST Accel. Beams 1, 044201 (1998).

[2] M. Blaskiewicz and V. Ranjbar, Transverse beam transfer functions via the Vlasov equation, in Proceedings of NAPAC13, Pasadena, CA, USA (JACoW, Pasadena, 2013), p. 1427, http://accelconf.web.cern.ch/AccelConf/ PAC2013/papers/froaa2.pdf.

[3] M. Blaskiewicz, A. Chao, and Y. H. Chin, Understanding the effect of space charge on instabilities, in Proceedings of IPAC15, Richmond, VA, USA (JACoW, Richmond, 2015), p. 743, http://accelconf.web.cern.ch/AccelConf/IPAC2015/ papers/mopmn019.pdf.

[4] A. Burov, Head-tail modes for strong space charge, Phys. Rev. ST Accel. Beams 12, 044202 (2009); 12, 109901(E) (2009).

[5] V. Balbekov, Transverse modes of a bunched beam with space charge dominated impedance, Phys. Rev. ST Accel. Beams 12, 124402 (2009).

[6] V. Kornilov, O. Boine-Frankenheim, and I. Hofmann, Stability of transverse dipole modes in coasting ion beams with nonlinear space charge, octupoles, and chromaticity, Phys. Rev. ST Accel. Beams 11, 014201 (2008).

[7] V. Kornilov and O. Boine-Frankenheim, Head-tail instability and Landau damping in bunches with space charge, Phys. Rev. ST Accel. Beams 13, 114201 (2010).

[8] V. Kornilov and O. Boine-Frankenheim, Transverse decoherence and coherent spectra in long bunches with space charge, Phys. Rev. ST Accel. Beams 15, 114201 (2012).

[9] A. Macridi, A. Burov, E. Stern, J. Amundson, and P. Spentzouris, Simulation of transverse modes with their intrinsic Landau damping for bunched beams in the presence of space charge, Phys. Rev. ST Accel. Beams 18, 074401 (2015).

[10] E. Metral and F. Ruggiero, Report No. CERN-AB-2004025 (ABP), 2004.

[11] K. Y. Ng, Report No. FERMILAB-CONF-08-410-AD, 2008.

[12] Y. H. Chin and K. Satoh, Transverse mode coupling in a bunched beam, IEEE Trans. Nucl. Sci. 30, 2566 (1983); Nucl. Instrum. Methods Phys. Res. 207, 309 (1983).

[13] A. Chao, Physics of Collective Beam Instabilities in High Energy Accelerators (John Wiley \& Sons, Inc., New York, 1993).

[14] K. Y. Ng and A. V. Burov, Report No. FERMILAB-FN685, 1999. 